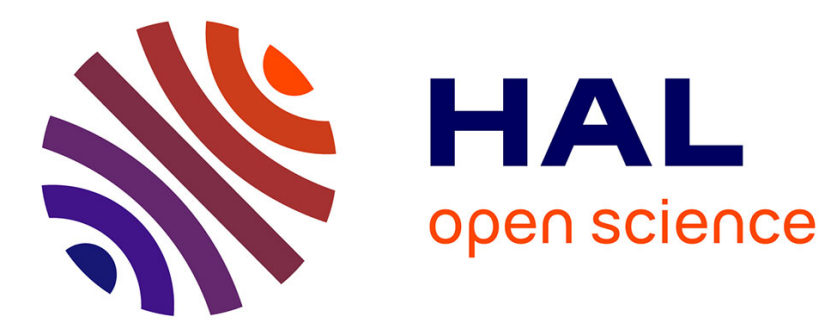

\title{
Mobility-aware Scheduler in CoMP Systems
}

Nivine Abbas, Thomas Bonald, Berna Sayrac

\section{To cite this version:}

Nivine Abbas, Thomas Bonald, Berna Sayrac. Mobility-aware Scheduler in CoMP Systems. PIMRC,

Sep 2016, Valencia, Spain. hal-01342029

\section{HAL Id: hal-01342029 \\ https://hal.science/hal-01342029}

Submitted on 5 Jul 2016

HAL is a multi-disciplinary open access archive for the deposit and dissemination of scientific research documents, whether they are published or not. The documents may come from teaching and research institutions in France or abroad, or from public or private research centers.
L'archive ouverte pluridisciplinaire $\mathbf{H A L}$, est destinée au dépôt et à la diffusion de documents scientifiques de niveau recherche, publiés ou non, émanant des établissements d'enseignement et de recherche français ou étrangers, des laboratoires publics ou privés. 


\section{Mobility-aware Scheduler in CoMP Systems}

\author{
Nivine Abbas \\ Orange Labs and Télécom ParisTech, \\ Université Paris-Saclay, France \\ nivine.abbas@telecom-paristech.fr
}

\author{
Thomas Bonald \\ Télécom ParisTech, \\ Université Paris-Saclay, France \\ thomas.bonald@telecom-paristech.fr
}

\author{
Berna Sayrac \\ Orange Labs \\ Châtillon, France \\ berna.sayrac@orange.com
}

\begin{abstract}
The main weakness of coordination techniques in LTE-Advanced networks is the extra resource consumption incurred by the joint transmission from several base stations. In this paper, we propose a new scheduling policy that performs coordination primarily for users staying at the cell edge, without mobility. Other cell-edge users are likely to move and to be served in better radio conditions where cell coordination is not required. We compare the performance of this algorithm to other usual scheduling policies in the presence of elastic traffic through the analysis of flow-level traffic models.
\end{abstract}

Index Terms-Cellular data networks, mobility, sector coordination, flow-level modeling, queuing theory.

\section{INTRODUCTION}

Inter-cell interference is one of the key challenges faced in mobile communication systems. It restricts the re-usability of the radio resource and limits spectral efficiency. Since the days of GSM, various techniques are used to cope with interference. Coordinated MultiPoint (CoMP) [1], [2], a main feature on the LTE-A roadmap, is mentioned as a promising approach to mitigate its effects through the coordination of multiple cells.

CoMP allows a group of eNodeBs to cooperate in order to improve the coverage, cell-edge throughput, and system efficiency. These eNodeBs, referred to as cooperating eNodeBs, communicate together through the backhaul network. This paper focuses on joint transmission (JT) scheme, where all cells of the coordination cluster are involved in the transmission and thus not available for other users. JT scheme has been demonstrated as an efficient approach to improve cell-edge user's throughput especially in high interference environments but this is at the cost of higher resource consumption. Thus there is a tradeoff between the performance of cell-edge users and the ability of the network to process all traffic. It is well known that CoMP schemes are only advantageous for cell-edge users [3]. It has recently been shown that in a low interference environment they can also be detrimental for celledge users at high load, due to the inefficient utilization of radio resources [4]. Their efficiency in fact depends critically on the scheduling strategy. It is proposed in [5], [6] to allocate a dedicated frequency band to cell-edge users in order to perform CoMP operations. In [7], the authors propose a joint proportional fairness scheduling algorithm that treats cellcenter and cell-edge users equally without any frequency band partitioning. The scheduling algorithm may also depend on

Nivine Abbas and Thomas Bonald are members of LINCS, see www.lincs.fr. the clustering technique, from static clusters with centralized scheduling [8] to dynamic clusters with distributed scheduling [9]. However, it was common in these works to assume static or semi static users in the performance evaluation of CoMP schemes.

Mobility is generally thought as improving throughput performance in the presence of elastic traffic, see for instance [10], [11], [12], [13]. However, it was shown in [14] that mobility has a critical impact on the performance of CoMP schemes. Indeed, in the presence of coordination mechanisms mobility can lead to bad performance if the scheduling strategy is not well chosen. Deprioritizing CoMP users seems to be a good strategy if they are able to move and to be served in good radio conditions without performing coordination.

Motivated by the above observation, we propose in this paper a mobility-aware scheduler that exploits the mobility as an additional information in order to schedule users with elastic traffic on the downlink of cellular data networks. This may require an estimation of the mobile speed as done in [15], [16]. Specifically, users requiring coordination are deprioritized if they are moving, so they are likely to be served in better radio conditions where cell coordination is not required avoiding the extra resource consumption incurred by the joint transmission from several base stations. We first study the case of one mobility behavior so that users are either static or mobile. We derive in each case the stability conditions. Then in order to evaluate the proposed scheduler we consider the case of several mobility behaviors. We show that this scheduler improves the global performance in this case.

The rest of the paper is organized as follows. In the next section, we describe the mobility-aware scheduler. Then we discuss various scheduling schemes in the absence of mobility in Section III. We analyze the impact of mobility in Section IV. We then extend the model to the case of several mobility behaviors in order to evaluate the mobility-aware scheduler in Section V. Section VI concludes the paper.

\section{Mobility-AWARE SCHEDULER}

When at the cell-edge, a user is able to receive signals from multiple cells and the user's transmission can be received at multiple cells, so if the signaling transmitted from those multiple cells is coordinated, the downlink performance can be improved significantly. This is the main idea of inter-cell coordination. Hence when a cell is involved in a coordination process, its resources are shared between its users and the 
additional users from neighboring cells which require the cooperation of the considered cell to be served. Thus the scheduling strategy is a key component of cellular systems supporting inter-cell coordination as the scheduling decision of a cell may affect the decisions of all its cooperating cells.

We consider in this paper a centralized scheduler in each cluster, that is the set of coordinated cells. This scheduler has a global view of all the users in the given cluster and thus can make decisions about the scheduled users of all the coordinated cells that belong to the cluster. The scheduling strategy can either be a fair strategy that treats CoMP and non-CoMP users equally or a prioritization strategy that prioritizes one category of users. We shall see in the following sections that the performance of the scheduling scheme depends primarily on users' mobility.

Deprioritizing CoMP users in the presence of mobility gives them time to move so that they are more likely served in good radio conditions where cell coordination is not required and thus improves performance. However this strategy decreases the system stability condition when users are static, since the condition of serving a CoMP user is more restrictive than that of serving a non-CoMP user due to the fact that it requires the availability of all coordinated cells and thus static CoMP users are never served at high load. It turns out that in a system with multiple mobility behaviors, static CoMP users should be treated equally as non-CoMP users, while it is better to deprioritize mobile CoMP users; the more the CoMP user is mobile the more it should be deprioritized. This can be done by assigning a score inversely proportional to the speed to each CoMP users, so that the scheduling algorithm becomes mobility-aware. For instance, the score is equal to one for static CoMP users and less than one for mobile CoMP users. The algorithm works as described below in Algorithm 1:

\section{Model Without Mobility}

We present in this section the reference model in the absence of mobility under various scheduling schemes.

\section{A. Cellular network}

In order to study the intra-site joint transmission technique, we consider a site constituted of $K=3$ sectors, where each sector $k=1,2,3$ is modeled by two main zones: a noncoordination zone and a coordination zone. We refer by zone $k, k+1$ (with modulo $\mathrm{K}$ notation) to the coordination zone between sectors $k$ and $k+1$. The coordination zones $k, k+1$ (hatched area), as illustrated by Figure 1, are those where the difference between the signals received from sectors $k$ and $k+1$ doesn't exceed a given threshold $\delta P$, involving both sectors in the transmission. We refer by zone $k$ to the noncoordination zone (area without hatching) where only sector $k$ is involved in the transmission.

We model each zone $k$ and zone $k, k+1$ by a set of $N_{k}$ and $N_{k k+1}$ regions respectively. In each region, radio conditions are supposed to be homogeneous and thus users are served at the same physical data rate on the downlink. We model each region by a queue with a specific service rate corresponding to

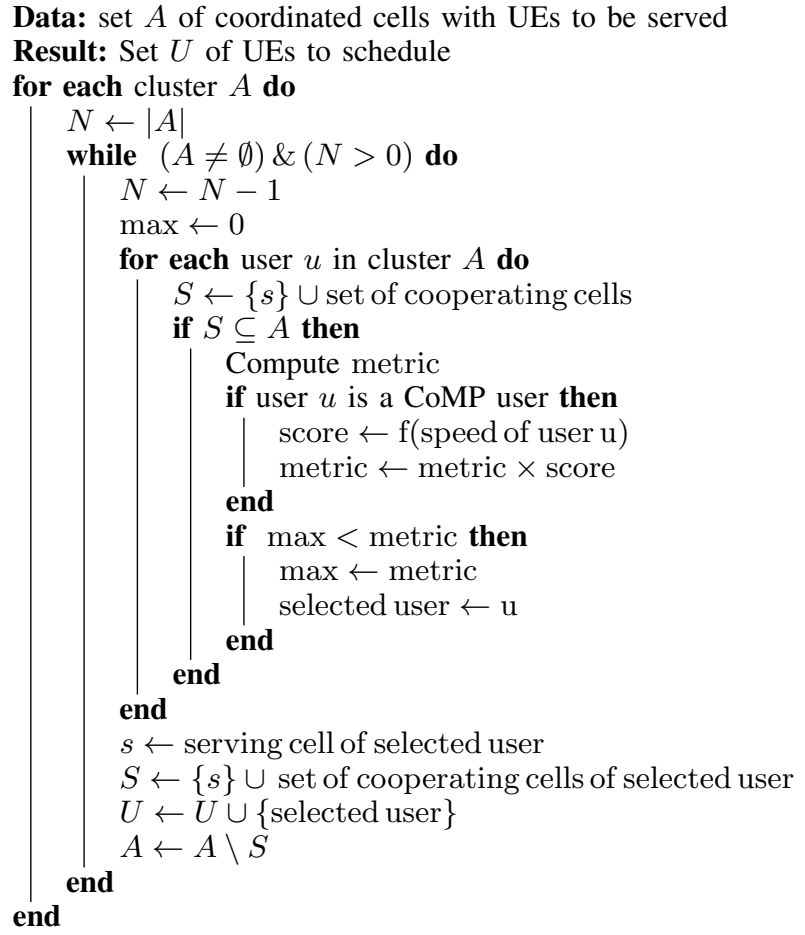

Algorithm 1: Mobility-aware algorithm.

the physical data rate in this region. The considered site shown in Figure 1 can be viewed as a set of $\sum_{k=1}^{K}\left(N_{k}+N_{k k+1}\right)$ queues with $K$ coupled processors.

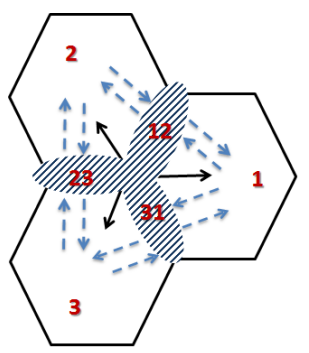

Fig. 1. A tri-sector site with coordination areas.

\section{B. Traffic model}

We consider elastic traffic only and we assume that new data flows are generated in region $i$ of zone $k$ and region $i$ of zone $k, k+1$ at the random times of a Poisson process of intensity $\lambda_{k}^{(i)}$ and $\lambda_{k, k+1}^{(i)}$ respectively. We denote by $\lambda_{k}=\sum_{i} \lambda_{k}^{(i)}$ and $\lambda_{k, k+1}=\sum_{i} \lambda_{k, k+1}^{(i)}$ the total flow arrival rates in zone $k$ and zone $k, k+1$ respectively. Let $\lambda=\sum_{k}\left(\lambda_{k}+\lambda_{k, k+1}\right)$ be the total flow arrival rate in the site. Thus, the probability that a new data flow is generated by a user in zone $k$ is $p_{k}=$ $\lambda_{k} / \lambda$ while the probability that a new data flow in zone $k$ is generated by a user in region $i$ is $p_{k}^{(i)}=\lambda_{k}^{(i)} / \lambda_{k}$. Similarly, the probability that a new data flow is generated by a user in zone $k, k+1$ is $p_{k, k+1}=\lambda_{k, k+1} / \lambda$ while the probability that a new data flow in zone $k, k+1$ is generated by a user in region $i$ is $p_{k, k+1}^{(i)}=\lambda_{k, k+1}^{(i)} / \lambda_{k, k+1}$. Each data flow is viewed 
as a fluid of random volume of exponential distribution with mean $\sigma$ (in bits) to be transmitted. When region $i$ of zone $k$ is served, flows are completed at rate $\mu_{k}^{(i)}$ in the absence of fast fading, corresponding to the physical rate $\mu_{k}^{(i)} \sigma$ (in bit/s). The service rate in region $i$ of zone $k, k+1$ is $\mu_{k, k+1}^{(i)}$.

We denote by $X_{k}(t)=\sum_{i} X_{k}^{(i)}(t)$ the total number of active flows in zone $k$ at time $t$ and by $X_{k, k+1}(t)=$ $\sum_{i} X_{k, k+1}^{(i)}(t)$ the total number of active flows in zone $k, k+1$, where $X_{k}^{(i)}(t)$ and $X_{k, k+1}^{(i)}(t)$ are the total numbers of active flows in region $i$ of zone $k$ and region $i$ of zone $k, k+1$ respectively. The vector $X(t)=\left(X_{k}^{(i)}(t), X_{k, k+1}^{(j)}(t)\right)_{(k, i, j)}$ defines a Markov process of dimension $\sum_{k} N_{k}+N_{k, k+1}$. The load of region $i$ in zone $k$ is $\rho_{k}^{(i)}=\lambda_{k}^{(i)} / \mu_{k}^{(i)}$, while the load of region $i$ in zone $k, k+1$ is $\rho_{k, k+1}^{(i)}=\lambda_{k, k+1}^{(i)} / \mu_{k, k+1}^{(i)}$. The total load of zone $k$ is given by $\rho_{k}=\sum_{i} \rho_{k}^{(i)}=\lambda_{k} / \mu_{k}$ and that of zone $k, k+1$ is given by $\rho_{k, k+1}=\sum_{i} \rho_{k, k+1}^{(i)}=\lambda_{k, k+1} / \mu_{k, k+1}$, where $\mu_{k}$ and $\mu_{k, k+1}$ are the weighted harmonic mean service rates:

$$
\mu_{k}=\frac{1}{\sum_{i} p_{k}^{(i)} / \mu_{k}^{(i)}} \text { and } \mu_{k, k+1}=\frac{1}{\sum_{i} p_{k, k+1}^{(i)} / \mu_{k, k+1}^{(i)}} .
$$

The resources of each sector $k$ are shared between CoMP users in coordination zones $k, k+1$ and $k-1, k$ and non-CoMP users in the non-coordination zone $k$. The actual service rate in zone $k$ is modulated by $\phi_{k}$ while $\phi_{k, k+1}$ represents the actual service rate in zone $k, k+1 . \phi_{k}$ and $\phi_{k, k+1}$ are the fractions of time spent by the scheduler on users in zone $k$ and zone $k, k+1$ respectively. This depends on the system state $x$ and on the scheduling policy. For work-conserving policies, we have

$$
\forall k: \quad \phi_{k}(x)+\phi_{k, k+1}(x)+\phi_{k-1, k}(x)=1 .
$$

For the sake of simplicity, we assume that users in each zone share the allocated radio resources equally, independently of their radio conditions. When a user in a coordination zone $k, k+1$ is served, all users in all coordination zones are blocked and thus,

$$
\sum_{k=1}^{K} \phi_{k, k+1}(x) \leq 1
$$

We study different resource sharing strategies between CoMP and non-CoMP users. Note that when a user in a coordination zone $k, k+1$ is served, users in non-coordination zones $k$ and $k+1$ are blocked and vice versa.

While the sufficient stability conditions depend on the scheduling policy, the necessary stability conditions are given by:

$$
\left\{\begin{array}{l}
\forall k: \quad \rho_{k}+\rho_{k, k+1}+\rho_{k-1, k}<1 \\
\sum_{k=1}^{K} \rho_{k, k+1} \leq 1
\end{array}\right.
$$

Let

$\rho=\lambda / \mu=\max \left(\max _{k}\left(\rho_{k}+\rho_{k, k+1}+\rho_{k-1, k}\right), \sum_{k=1}^{K} \rho_{k, k+1}\right)$.
We shall see in the following sections that mobility may increase or decrease the stability condition, depending on the scheduling strategy.

\section{Throughput metrics}

We measure performance in terms of mean throughputs in the different zones. In any state $x$ such that $x_{k}^{(i)}>0$, each user in region $i$ of zone $k$ has throughput $\mu_{k}^{(i)} \sigma \phi_{k}(x) / x_{k}$. We denote by $E_{k}^{(i)}$ and $E_{k, k+1}^{(i)}$ the expectations corresponding to the following size-biased distributions [17]:

$\pi_{k}^{(i)}(x) \propto x_{k}^{(i)} \pi(x)$ and $\pi_{k, k+1}^{(i)} \propto x_{k, k+1}^{(i)} \pi(x)$.

The mean throughput of users in zone $k$ is then given by

$$
\gamma_{k}=E\left(\sum_{i} \mu_{k}^{(i)} \sigma \phi_{k}(X) \frac{X_{k}^{(i)}}{X_{k}}\right) / E\left(X_{k}\right) \text {. }
$$

This is the arithmetic mean of the mean throughputs $\gamma_{k}^{(i)}$ weighted by the probabilities $p_{k}^{\prime(i)}$, where

$$
p_{k}^{\prime(i)}=E\left(X_{k}^{(i)}\right) / E\left(X_{k}\right) .
$$

Similarly, the mean throughput in zone $k, k+1$ is given by

$$
\gamma_{k, k+1}=E\left(\sum_{i} \mu_{k, k+1}^{(i)} \sigma \phi_{k, k+1}(X) \frac{X_{k, k+1}^{(i)}}{X_{k, k+1}}\right) / E\left(X_{k, k+1}\right) \text {. }
$$

The mean throughput in the site is the arithmetic mean of the mean throughputs $\gamma_{k}$ (4) and $\gamma_{k, k+1}$ (5) weighted by the probabilities $p_{k}^{\prime}$ and $p_{k, k+1}^{\prime}$ respectively, given by $p_{k}^{\prime}=\frac{E\left(X_{k}\right)}{\sum_{j=1}^{K} E\left(X_{j}\right)+E\left(X_{j, j+1}\right)}$ and $p_{k, k+1}^{\prime}=\frac{E\left(X_{k, k+1}\right)}{\sum_{j=1}^{K} E\left(X_{j}\right)+E\left(X_{j, j+1}\right)}$.

\section{Static allocation}

A simple strategy consists in allocating a fixed fraction of resources to CoMP and non-CoMP users' data flows, as proposed by 3GPP in [5], [6]. CoMP users' data flows are allocated some fixed fraction $\phi_{0}$ of the resources of each coordinated sector. In particular, CoMP users' data flows in zone $k, k+1$ are allocated the fraction:

$$
\phi_{k, k+1}=\frac{x_{k, k+1}}{\sum_{j=1}^{K} x_{j, j+1}} \phi_{0} .
$$

Non-CoMP users' data flows in sector $k$ are allocated the fraction $\phi_{k}=1-\phi_{0}$ of the considered sector radio resources. From (4) and (5), the mean throughput in zone $k$ is

$$
\gamma_{k}=\phi_{k} \mu_{k} \sigma\left(1-\rho_{k} / \phi_{k}\right) \quad k=1 \ldots K
$$

while the mean throughput in zone $k, k+1$ is:

$$
\gamma_{k, k+1}=\phi_{0} \mu_{k, k+1} \sigma\left(1-\sum_{j=1}^{K} \rho_{j, j+1} / \phi_{0}\right) \text {. }
$$

Observe that the mean throughput in zone $k$ decreases linearly from the physical rate $\phi_{k} \mu_{k} \sigma$ to 0 when the load of the corresponding zone increases from 0 to $\phi_{k}$. Indeed, the stability 
condition of zone $k$ is $\rho_{k}<\phi_{k}$. The stability condition is given by:

$$
\left\{\begin{array}{l}
\forall k: \quad \rho_{k}<1-\phi_{0} \\
\sum_{k=1}^{K} \rho_{k, k+1}<\phi_{0}
\end{array}\right.
$$

Observe that this condition may be more restrictive than the natural stability condition (3).

\section{E. Iterative scheduler}

Under the iterative scheduler [4], users in zone $k$ are allocated the fraction

$$
\begin{gathered}
\phi_{k}(x)=\frac{x_{k}+x_{k+1, k-1}}{\bar{x}} \\
+\frac{x_{k+1}}{\bar{x}} \frac{x_{k}+x_{k-1}}{x_{k}+x_{k-1, k}+x_{k-1}}+\frac{x_{k-1}}{\bar{x}} \frac{x_{k}+x_{k+1}}{x_{k}+x_{k, k+1}+x_{k+1}}
\end{gathered}
$$

of sector $k$ resources while users in zone $k, k+1$ are allocated the fraction

$$
\phi_{k, k+1}(x)=\frac{x_{k, k+1}}{\bar{x}}+\frac{x_{k-1}}{\bar{x}} \frac{x_{k, k+1}}{x_{k}+x_{k, k+1}+x_{k+1}}
$$

of each coordinated sector. We denote by

$$
\bar{x}=\sum_{k=1}^{K}\left(x_{k}+x_{k, k+1}\right)
$$

the total number of active users in the site. Indeed, a centralized scheduler should be implemented in each site. For instance, if a user in zone 1 is selected to be served by the scheduling algorithm, the scheduler can either select a user in zone 2 and a user in zone 3 or select a user in zone 23 to be served simultaneously. Similarly, if a user in zone 12 is selected for instance, another user in zone 3 is served simultaneously. The stability condition is given by (3). When

$$
\max _{k}\left(\rho_{k}+\rho_{k, k+1}+\rho_{k-1, k}\right)>\sum_{k=1}^{K} \rho_{k, k+1},
$$

explicit expressions for the flow throughputs can be obtained by approximation. We proceed by decoupling the different zones as in [4]. We consider zone $k$, zone $k+1$ and zone $k, k+1$ and neglect the other zones after taking into account the load induced by zone $k-1, k$ on cell $k$ and that induced by zone $k+1, k-1$ on cell $k+1$. Thus the flow throughput in zone $k$ is approximately:

$$
\gamma_{k} \approx \mu_{k} \sigma\left(1-\rho_{k}-\rho_{k, k+1}-\rho_{k-1, k}\right)
$$

while the flow throughput in zone $k, k+1$ is approximately:

$$
\begin{gathered}
\gamma_{k, k+1} \approx \mu_{k, k+1} \sigma \\
\left(\sum_{j=k}^{k+1} \frac{1}{1-\rho_{j, j+1}-\rho_{j-1, j}-\rho_{j}}-\frac{1}{1-\rho_{k, k+1}}\right)^{-1} .
\end{gathered}
$$

\section{F. Priority to non-CoMP users}

Under this policy, non-CoMP users in each sector are scheduled first and are allocated all the radio resources whenever active: in any state $x$ such that $x_{k}>0 \phi_{k}=1$, that is

$$
\bar{\phi}_{k}=\rho_{k} .
$$

CoMP users in zone $k, k+1$ wait until resources in zones $k$ and $k+1$ become available, these users equally share resources with other CoMP users in the other coordination zones. The fraction of time spent by the scheduler on users in zone $k, k+1$ is:

$\phi_{k, k+1}= \begin{cases}\frac{x_{k, k+1}}{\sum_{j=1}^{K} x_{j, j+1}} & \text { if } \sum_{k=1}^{K} x_{k}=0 . \\ 1 & \text { if } \sum_{k=1}^{K} x_{k}>0 \& x_{k}+x_{k+1}=0 . \\ 0 & \text { otherwise. }\end{cases}$

Thus the mean throughput in non-coordination zone $k$ is:

$$
\gamma_{k}=\mu_{k} \sigma\left(1-\rho_{k}\right) .
$$

However there is no explicit expression for the mean throughput in the coordination zones. The stability condition is given by:

$\forall k: \rho_{k, k+1}<\left(1-\rho_{k}\right)\left(1-\rho_{k+1}\right)\left(\rho_{k-1}+\left(1-\rho_{k-1}\right) \theta_{k, k+1}\right)$ such that $\sum_{k=1}^{K} \theta_{k, k+1}<1$, we get

$$
\sum_{k=1}^{K}\left(\rho_{k, k+1}-\rho_{k} \prod_{j \neq k}\left(1-\rho_{j}\right)\right)<\prod_{k=1}^{K}\left(1-\rho_{k}\right),
$$

which is more restrictive than the natural stability condition (3). This is due to the fact that some cells may be idle when serving non-CoMP users.

\section{G. Priority to CoMP users}

Under this policy, CoMP users are scheduled first and are allocated all radio resources whenever active: users in zone $k, k+1$ are allocated the fraction:

$$
\phi_{k, k+1}=x_{k, k+1} / \sum_{j=1}^{K} x_{j, j+1} .
$$

Non-CoMP users in zone $k$ are served only when there are no active CoMP users in zones $k, k+1$ and $k-1, k$. The stability condition is the natural condition given by (3). Since CoMP users are not affected by non-CoMP users the mean throughput in the coordination zone $k, k+1$ is given by: $\gamma_{k, k+1}=\mu_{k, k+1} \sigma\left(1-\sum_{j=1}^{K} \rho_{j, j+1}\right)$.

\section{H. Numerical results}

We consider the case of $\delta P=12 d B$ in a beamforming system where the mean coordination gain is around $50 \%$. In this case, the mean coordination surface of each sector is around $30 \%$, that is $p_{1}=p_{2}=p_{3}=7 / 30$ and $p_{12}=p_{13}=p_{23}=1 / 10$. Note that this case corresponds to a low interference scenario that is a scenario with a moderate mean coordination gain. We assume that there is only one region in each zone with $\mu_{1}=\mu_{2}=\mu_{3}=2$ and 
$\mu_{12}=\mu_{13}=\mu_{23}=1.5$ and we consider $\phi_{0}=1 / 2$ for the static allocation (SA). Figure 2 (dashed lines) compares the throughput performance of the four policies. Observe that the iterative scheduler (IT) outperforms the three other strategies. The priority to non-CoMP (Pri-NC) strategy leads to degraded performance. These results show that in the absence of mobility a fair strategy is the best strategy to be applied. The maximum load is approximately equal to 0.83 under the Pri$\mathrm{NC}$ scheme and to 0.625 under the SA scheme, in accordance with (6) and (7).

\section{IMPACT OF MOBILITY}

In this section, we add mobility to the previous model. We consider only intra-site mobility as inter-site mobility will have only limited impact on the performance, similarly to handovers considered in [10]. We suppose that users can move from one region to another as well as from one zone to another zone.

\section{A. Mobility model}

1) Inter-zone mobility: In a general case we assume that each user in region $i$ of non-coordination zone $k$ moves to region $n$ of a non-coordination zone $j$ and vice versa after exponential durations, at respective rates $\nu_{k \rightarrow j}^{(i, n)}$ and $\nu_{j \rightarrow k}^{(n, i)}$. In state $x$, the total mobility rate from region $i$ of zone $k$ to region $n$ of zone $j$ and from region $n$ of zone $j$ to region $i$ of zone $k$ are equal to $x_{k}^{(i)} \nu_{k \rightarrow j}^{(i, n)}$ and $x_{j}^{(n)} \nu_{j \rightarrow k}^{(n, i)}$ respectively. Similarly, the total mobility rate from region $i$ of a non-coordination zone $k$ to region $n$ of a coordination zone $j, j+1$ and from region $n$ of zone $j, j+1$ to region $i$ of zone $k$ are equal to $x_{k}^{(i)} \nu_{k \rightarrow j, j+1}^{(i, n)}$ and $x_{j, j+1}^{(n)} \nu_{j, j+1 \rightarrow k}^{(n, i)}$ respectively. We assume that users can also move from a coordination zone $k, k+1$ to another coordination zone $j, j+1$. Thus the total mobility rate is equal to $x_{k, k+1}^{(i)} \nu_{k, k+1 \rightarrow j, j+1}^{(i, n)}$.

2) Intra-zone mobility: We assume that in each zone $k$, each user in region $i$ moves to region $n \neq i$ (for $i, n \in$ $\left.\left\{1 \ldots N_{k}\right\}\right)$ after exponential durations, at rate $\nu_{k}^{(i, n)}$. The total mobility rate from region $i$ to region $n$ is $x_{k}^{(i)} \nu_{k}^{(i, n)}$.

At high load, the mobility process can be viewed as a Markov process of $\sum_{k=1}^{K}\left(N_{k}+N_{k, k+1}\right)$ states, where each state represents a region of a given zone. The transitions between the different sates are equal to the corresponding mobility rates. The probability that a user is in region $i$ of zone $k$ is $q_{k}^{(i)}$ while the probability that a user is in region $i$ of zone $k, k+1$ is $q_{k, k+1}^{(i)}$. These probabilities are given by the stationary distribution and follow from the following balance equations

$$
\begin{gathered}
\forall(k, i): \\
q_{k}^{(i)}\left(\sum_{\substack{n \leq N_{k} \\
n \neq i}} \nu_{k}^{(i, n)}+\sum_{\substack{n \leq N_{j} \\
j \neq k}} \nu_{k \rightarrow j}^{(i, n)}+\sum_{\substack{n \leq N_{j, j+1} \\
j \leq K}} \nu_{k \rightarrow j, j+1}^{(i, n)}\right) \\
=\sum_{\substack{n \leq N_{k} \\
n \neq i}} \nu_{k}^{(n, i)} q_{k}^{(n)}+\sum_{\substack{n \leq N_{j} \\
j \neq k}} \nu_{j \rightarrow k}^{(n, i)} q_{j}^{(n)}+\sum_{\substack{n \leq N_{j, j+1} \\
j \leq K}} \nu_{j, j+1 \rightarrow k}^{(n, i)} q_{j, j+1}^{(n)}
\end{gathered}
$$

$$
\begin{gathered}
\text { and } q_{k, k+1}^{(i)} \times \\
\left(\sum_{\substack{n \leq N_{k, k+1} \\
n \neq i}} \nu_{k, k+1}^{(i, n)}+\sum_{\substack{n \leq N_{j} \\
j \leq K}} \nu_{k, k+1 \rightarrow j}^{(i, n)}+\sum_{\substack{n \leq N_{j, j+1} \\
j \neq k}} \nu_{k, k+1 \rightarrow j, j+1}^{(i, n)}\right) \\
=\sum_{\substack{n \leq N_{k, k+1} \\
n \neq i}} \nu_{\substack{k, k+1 \\
k, k+1}}^{(n)} q_{k, k+1}^{(n)}+\sum_{\substack{n \leq N_{j} \\
j \leq K}} \nu_{j \rightarrow k, k+1}^{(n, i)} q_{j}^{(n)} \nu_{\substack{n \leq N_{j, j+1} \\
j \neq k}}^{(n, i)} \\
+\sum_{j, j+1 \rightarrow k, k+1}^{(n)} q_{j, j+1},
\end{gathered}
$$

With

$$
\sum_{k=1}^{K}\left(\sum_{i=1}^{N_{k}} q_{k}^{(i)}+\sum_{n=1}^{N_{k, k+1}} q_{k, k+1}^{(n)}\right)=1
$$

The probability that a user is in zone $k$ is then given by: $q_{k}=\sum_{i=1}^{N_{k}} q_{k}^{(i)}$, while the probability that a user is in zone $k, k+1$ is: $q_{k, k+1}=\sum_{i=1}^{N_{k, k+1}} q_{k, k+1}^{(i)}$. We denote by $q_{0}$ the probability that a user is in a coordination zone, that is: $q_{0}=\sum_{k=1}^{K} q_{k, k+1}$.

\section{B. Stability condition}

The stability condition follows from the limiting regime of infinite mobility where:

$\left\{\begin{array}{l}\forall(k, j, i, n): \nu_{k \rightarrow j, j+1}^{(i, n)}, \nu_{j, j+1 \rightarrow k}^{(n, i)}, \nu_{k \rightarrow j}^{(i, n)}, \nu_{k, k+1 \rightarrow j, j+1}^{(i, n)} \rightarrow \infty \\ \forall(k, i, n): \quad \nu_{k}^{(i, n)} \rightarrow \infty\end{array}\right.$

In this regime the mean service rate in zone $k$ becomes

$$
\bar{\mu}_{k}=\sum_{i=1}^{N_{k}} q_{k}^{(i)} \mu_{k}^{(i)} / q_{k}
$$

Similarly, the mean service rate in zone $k, k+1$ is:

$$
\bar{\mu}_{k, k+1}=\sum_{i=1}^{N_{k, k+1}} q_{k, k+1}^{(i)} \mu_{k}^{(i)} / q_{k, k+1}
$$

Considering only intra-zone mobility without inter-zone mobility, the stability condition is:

$$
\left\{\begin{array}{c}
\forall k: \quad \begin{array}{r}
\mu_{k} \rho_{k} / \bar{\mu}_{k}+\mu_{k, k+1} \rho_{k, k+1} / \bar{\mu}_{k, k+1} \\
+\mu_{k-1, k} \rho_{k-1, k} / \bar{\mu}_{k-1, k}<1
\end{array} \\
\sum_{k=1}^{K} \mu_{k, k+1} \rho_{k, k+1} / \bar{\mu}_{k, k+1} \leq 1
\end{array}\right.
$$

under the IT and Pri-C schemes,

$$
\left\{\begin{array}{l}
\forall k: \quad \mu_{k} \rho_{k} / \bar{\mu}_{k}<1-\phi_{0} \\
\sum_{k=1}^{K} \mu_{k, k+1} \rho_{k, k+1} / \bar{\mu}_{k, k+1} /<\phi_{0}
\end{array}\right.
$$

under the SA scheme and

$$
\left.\sum_{k=1}^{K}\left(\frac{\mu_{k, k+1}}{\bar{\mu}_{k, k+1}} \rho_{k, k+1}-\frac{\mu_{k}}{\bar{\mu}_{k}} \rho_{k} \prod_{j \neq k}\left(1-\frac{\mu_{j}}{\bar{\mu}_{j}} \rho_{j}\right)\right)\right)
$$




$$
<\prod_{k=1}^{K}\left(1-\frac{\mu_{k}}{\bar{\mu}_{k}} \rho_{k}\right)
$$

under the Pri-NC scheme. However, in the presence of interzone mobility the stability condition becomes

$$
\rho<\bar{\mu} / \mu,
$$

where $\bar{\mu}$ is the overall mean service rate. The precise mean service rate depends on the scheduling strategy, where

$$
\bar{\mu}=\sum_{k=1}^{K}\left(1-\phi_{0}\right) \bar{\mu}_{k}+\phi_{0} \frac{q_{k, k+1}}{q_{0}} \bar{\mu}_{k, k+1}
$$

under the SA scheme,

$$
\bar{\mu}=\frac{1}{q_{0}} \sum_{k=1}^{K} q_{k, k+1}\left(\bar{\mu}_{k, k+1}+\bar{\mu}_{k-1}\right)
$$

under the Pri-C scheme,

$$
\bar{\mu}=\sum_{k=1}^{K} \bar{\mu}_{k}
$$

under the Pri-NC scheme and

$$
\bar{\mu}=\sum_{k=1}^{K} \phi_{k}(q) \bar{\mu}_{k}+\phi_{k, k+1}(q) \bar{\mu}_{k, k+1}
$$

under the IT scheduler. Note that

$$
\begin{gathered}
\phi_{k}(q)=q_{k}+q_{k+1, k-1} \\
+q_{k+1} \frac{q_{k}+q_{k-1}}{q_{k}+q_{k-1, k}+q_{k-1}}+q_{k-1} \frac{q_{k}+q_{k+1}}{q_{k}+q_{k, k+1}+q_{k+1}},
\end{gathered}
$$

while

$$
\phi_{k, k+1}(q)=q_{k, k+1}+q_{k-1} \frac{q_{k, k+1}}{q_{k}+q_{k, k+1}+q_{k+1}} .
$$

\section{Numerical results}

Consider the same scenario as in the previous section, with $\nu_{k-1, k \rightarrow k}=\nu_{k \rightarrow k, k+1}=1 \forall k$. All other mobility rates are supposed equal to zero. The results are obtained by the numerical evaluation of the stationary distribution of the Markov process $X(t)$ and shown in Figure 2 (solid lines). Inter-zone mobility improves the mean throughput in the site for all strategies except for the Pri-C strategy. Under this strategy, mobility leads to a throughput degradation. However, prioritizing non-CoMP users in the presence of mobility outperforms all other strategies in sharp contrast to the scenario without mobility. This is due to the fact that cell edge users are more likely served in good radio conditions without performing CoMP operations, minimizing the waste of resources. Based on this observation, the following conclusion may be drawn: in low coordination gain scenario, it is not worth losing a resource to serve a CoMP user if that user is moving and can benefit from better radio conditions. So if predicted accurately, mobility can be an interesting property which can include the scheduling strategy. Following this conclusion, we will introduce a more advanced mobility-aware scheduler in the next section.

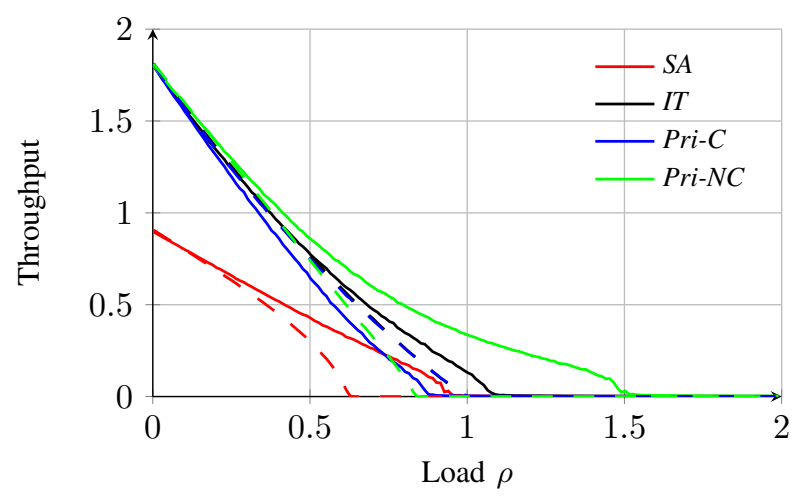

Fig. 2. Mean throughput in the site with mobility (solid line) and without mobility (dashed line).

\section{Multiple mobility BeHAViors}

Motivated by the above conclusions, we consider in this section the case of multiple mobility behaviors, so that users may be either static or mobile. Then we introduce a mobilityaware scheduler which uses the mobility as an additional information in order to schedule users. For instance, we assign to each CoMP user a score which is inversely proportional to its speed and which is used by the scheduler. The more the CoMP user is static the more it is prioritized.

\section{A. Multi-class extension}

We have assumed so far that all users have the same mobility behavior. In particular, they are all static or all mobile. In order to evaluate the mobility-aware scheduler, we extend the results to multiple classes of mobility, where each user belongs to a given class of mobility $v$, defined by a set of mobility rates

$$
\left(\nu_{k \rightarrow j, j+1}^{(i, n), v}, \nu_{j, j+1 \rightarrow k}^{(n, i), v}, \nu_{k \rightarrow j}^{(i, n), v}, \nu_{k, k+1 \rightarrow j, j+1}^{(i, n), v}, \nu_{k}^{(i, n), v}\right)_{(k, j, i, n)} .
$$

We consider $V$ classes of mobility. For instance, each class represents a range of speeds (static, pedestrian, users in train, etc). We assume mobility classes are numbered in decreasing order of speed so that $v=0$ represents the static class. We denote by $w_{v}$ the probability that a new flow is initiated by a class- $v$ user. The vector $X(t)=$ $\left(X_{k}^{(i), v}(t), X_{k, k+1}^{(n), v}(t)\right)_{(k, i, n, v)}$ defines a Markov process of dimension $V \times \sum_{k} N_{k}+N_{k, k+1}$. Let $X_{k}(t)=\sum_{v} X_{k}^{(v)}(t)$ and $X_{k, k+1}(t)=\sum_{v} X_{k, k+1}^{(v)}(t)$, where $X_{k}^{(v)}(t)=$ $\sum_{i \leq N_{k}} X_{k}^{(i), v}(t)$ and $X_{k, k+1}^{(v)}(t)=\sum_{i \leq N_{k, k+1}} X_{k, k+1}^{(i), v}(t)$ are the total numbers of class- $v$ users in zone $k$ and zone $k, k+1$ respectively.

\section{B. Mobility-aware scheduling}

Under the mobility-aware scheduler, non-CoMP users and static CoMP users are served first and then mobile CoMP users who are served in the increasing order of speed. Users in zone $k$ are allocated the fraction:

$$
\phi_{k}(x)=\frac{x_{k}+x_{k+1, k-1}^{(0)}}{\bar{x}}
$$




$$
+\frac{x_{k+1}}{\bar{x}} \frac{x_{k}+x_{k-1}}{x_{k}+x_{k-1, k}^{(0)}+x_{k-1}}+\frac{x_{k-1}}{\bar{x}} \frac{x_{k}+x_{k+1}}{x_{k}+x_{k, k+1}^{(0)}+x_{k+1}}
$$

of sector $k$ resources. The share of class- $v$ non-CoMP users $(v \geq 0)$ is:

$$
\phi_{k}^{(v)}(x)=\frac{x_{k}^{(v)}}{x_{k}} \phi_{k}(x) .
$$

Class- $v$ CoMP users of zone $k, k+1$ are allocated the fraction

$$
\begin{array}{ll} 
& \phi_{k, k+1}^{(v)}(x)= \\
\frac{x_{k, k+1}^{(v)} \times x_{k-1} / \bar{x}}{x_{k}+x_{k, k+1}^{(v)}+x_{k+1}} & \text { if } v=0 \\
+\frac{x_{k, k+1}^{(v)}}{x} & \\
\frac{x_{k, k+1}^{(v)}}{\sum_{j} x_{j, j+1}^{(v)}} & \text { if } \begin{array}{l}
\sum_{j}\left(x_{j}+\sum_{s<v} x_{j, j+1}^{(s)}=0\right) \\
\end{array} \\
& \text { if } \begin{array}{l}
x_{k}+x_{k+1}+\sum_{s<v} x_{k, k+1}^{(s)}=0 \\
\phi_{k-1}(x)
\end{array} \\
& \text { otherwise. }
\end{array}
$$

of each coordinating sector (sector $k$ and sector $k+1$ ). Observe that the network operates under the IT scheduling strategy for the static users and under the Pri-NC users strategy for the mobile users. These shares of each class of users are used in order to compute the stationary distribution $\pi(x)$ of the Markov process $X(t)$, given the arrival rate, the service rate and the mobility rates in each region of each zone.

\section{Numerical results}

The numerical evaluation of the stationary distribution of the Markov process $X(t)$ is shown in Figure 3 for the same previous scenario but with two classes of users: a static class and a mobile class: $w_{0}=w_{1}=50 \%$. Results show that the

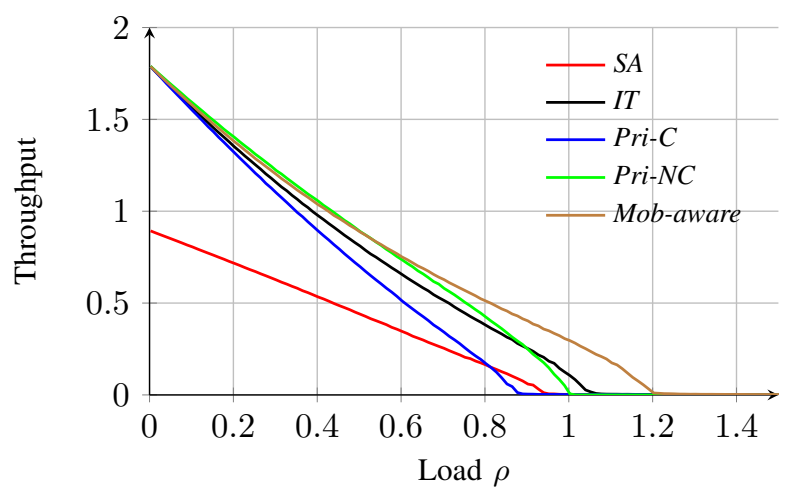

Fig. 3. Mean throughput in the site with two classes of mobility.

mobility aware scheduler improves the performance as well as the stability condition. Observe that there is an increase of approximately $15 \%$ of the stability condition compared to the IT strategy. The reason is that the proposed scheduler, by combining the IT (the best strategy for static users see Fig.2) and the Pri-NC (the best strategy for mobile users see Fig.2) strategies, can achieve the best stability condition for each mobility class.

\section{CONCLUSION}

In this paper we have shown that it is not worth to perform coordination in a low interference scenario for a mobile user who is able to move and to get better radio conditions. We have proposed a mobility-aware scheduler that deprioritize mobile users in bad radio conditions. Multi-point coordination is applied primarily to static users located at the cell edge. We have observed that the proposed scheduling strategy brings performance gains. This scheduler is suitable for elastic traffic for which delay is tolerable; we plan to address the issue of real-time traffic in future work. We aim also to validate the results by system level simulations.

\section{REFERENCES}

[1] 3GPP TR 36.913, "Requirements for Further Advancements for EUTRA (LTE-Advanced),

[2] R. Irmer, H. Droste, P. Marsch, M. Grieger, G. Fettweis, S. Brueck, H. Mayer, L. Thiele, and V. Jungnickel, "Coordinated multipoint: Concepts, performance, and field trial results," IEEE Communications Magazine, vol. 49, no. 2, pp. 102-111, 2011.

[3] 3GPP R1-083569, "Further Discussion on Inter-Cell Interference Mitigation Through Limited Coordination ," 2008.

[4] A. Khlass, T. Bonald, and S. Elayoubi, "Flow-level performance of intrasite coordination in cellular networks," in IEEE WiOpt, Tsukuba Japan, May 2013, pp. 216-223, 2013.

[5] 3GPP R1-090613, "Discussion on CoMP-SU-MIMO,” 2009.

[6] 3GPP R1-091415, "Further discussion of frequency plan scheme on CoMP-SU-MIMO," 2009.

[7] J. Liu, Y. Chang, Q. Pan, X. Zhang, and D. Yang, "A novel transmission scheme and scheduling algorithm for CoMP-SU-MIMO in LTE-A system," in Proceedings of the 71st IEEE VTC , 16-19 May 2010, Taipei, Taiwan, pp. 1-5, 2010.

[8] S. Brueck, L. Zhao, J. Giese, and M. A. Amin, "Centralized Scheduling for Joint Transmission Coordinated Multi-Point in LTE-Advanced," in Proc. ITG/IEEE Workshop on Smart Antennas, , 23-24 Feb 2010, Bremen, Germany, 2010.

[9] W. Zhou, Y. Zhang, P. Qin, W. Chen, and X. Li, "Joint scheduling algorithms for LTE-A comp system," JCP, vol. 8, no. 11, pp. 2795$2801,2013$.

[10] N. Abbas, T. Bonald, and B. Sayrac, "Opportunistic gains of mobility in cellular data networks," in IEEE WiOpt, Mumbai India, May 2015.

[11] T. Bonald, S. C. Borst, and A. Proutière, "How mobility impacts the flow-level performance of wireless data systems," in Proceedings IEEE INFOCOM 2004, Hong Kong, China, March 7-11, 2004.

[12] S. C. Borst, A. Proutière, and N. Hegde, "Capacity of wireless data networks with intra- and inter-cell mobility," in INFOCOM. 23-29 April 2006, Barcelona, Catalunya, Spain, 2006.

[13] S. C. Borst, N. Hegde, and A. Proutiere, "Mobility-driven scheduling in wireless networks," in INFOCOM. 19-25 April 2009, Rio de Janeiro, Brazil, pp. 1260-1268, 2009.

[14] N. Abbas, T. Bonald, and B. Sayrac, "How mobility impacts the performance of inter-cell coordination in cellular data networks," in IEEE Globecom, San Diego, CA, USA, December 2015.

[15] D.-G. Herculea, M. Haddad, V. Capdevielle, and C. Shue Chen, "Network-based UE mobility estimation in mobile networks," in $A C M$ MobiCom, Poster Paper, (Paris, France), Sep 2015.

[16] M. Haddad, D.-G. Herculea, E. Altman, N. Ben Rached, V. Capdevielle, C. Shue Chen, and F. Ratovelomanana, "Mobility State Estimation in LTE," in IEEE Wireless Communications and Networking Conference, (Doha, Qatar), IEEE, June 2016.

[17] T. Bonald and M. Feuillet, Network Performance Analysis. ISTE/Wiley, 2011. 